\title{
Effective inhibition of nasopharyngeal carcinoma in vitro and in vivo by targeting glycolysis with oxamate
}

\author{
XIAOBING LI ${ }^{1}$, WENHUA LU ${ }^{1}$, YUMIN HU ${ }^{1}$, SHIJUN WEN ${ }^{1}$, \\ CHAONAN QIAN $^{1}$, WENJING WU ${ }^{1}$ and PENG HUANG ${ }^{1,2}$ \\ ${ }^{1}$ State Key Laboratory of Oncology in South China, Sun Yat-Sen University Cancer Center, \\ Guangzhou 510275, P.R. China; ${ }^{2}$ Department of Translational Molecular Pathology, \\ The University of Texas M.D. Anderson Cancer Center, Houston, TX 77030, USA
}

Received June 14, 2013; Accepted July 19, 2013

DOI: 10.3892/ijo.2013.2080

\begin{abstract}
Elevated aerobic glycolysis in cancer cells (Warburg effect) has been observed in many tumor types including nasopharyngeal carcinoma (NPC), which can often be detected clinically using FDG-PET. However, the role of glycolysis in supporting the growth of NPC cells and its therapeutic implications still remain to be investigated. In the present study, we showed that the LDH inhibitor oxamate significantly suppressed NPC cell proliferation in vitro and tumor growth in vivo, yet exhibited minimum toxicity to normal nasopharyngeal epithelial cells in vitro and was well tolerated in mice. Moreover, oxamate exhibited cytotoxic effect in NPC cells under hypoxia. Mechanistic study showed that oxamate significantly inhibited LDH activity, leading to a substantial decrease in glucose uptake and lactate production. Combination of oxamate with a mitochondrial respiratory complex I inhibitor resulted in a significant depletion of cellular ATP and a synergistic killing of cancer cells. Our results suggest that inhibition of glycolysis by oxamate is an effective therapeutic strategy for treatment of NPC and that combination of this compound with mitochondrial-targeted agents may improve the therapeutic activity.
\end{abstract}

\section{Introduction}

Nasopharyngeal carcinoma (NPC) is a malignancy with unique geographic distribution and is most common in certain areas of Asia such as the southern part of China (1). In clinical practice, early detection of NPC still remains a major challenge and many NPC patients are diagnosed in the advanced stage with limited treatment options. This situation

Correspondence to: Dr Peng Huang, Department of Translational Molecular Pathology, The University of Texas M.D. Anderson Cancer Center, 1515 Holcombe Blvd, Houston, TX 77030, USA

E-mail: phuang@mdanderson.org

Key words: nasopharyngeal carcinoma, cancer metabolism, glycolysis, oxamate contributes to the relatively high mortality of NPC. Currently, the therapeutic options of NPC include chemotherapy, radiotherapy and chemoradiotherapy (2). Despite the significant progress in our understanding of NPC genetics and disease development, the therapy of NPC still remains to be a great challenge due to drug resistance, disease relapse and metastasis. The high toxicity of conventional chemotherapy and radiotherapy also affects the quality of life of NPC patients $(3,4)$. Therefore, it is of great importance to develop novel and effective therapeutic strategies that specifically kill NPC and is tolerated by normal cells (3). Metabolic intervention based on the unique metabolic alterations in cancer cells is one of the attractive therapeutic strategies with high anticancer activity and selectivity.

One of the major metabolic alterations in cancer cells is elevation in aerobic glycolysis, a phenomenon known as the Warburg effect observed many decades ago (5). The increase in glucose uptake by cancer cells constitute the key biochemical basis of ${ }^{18}$ fluorodeoxyglucose positron emission tomography (FDG-PET) for clinical diagnosis of cancer (6). Although the exact reasons for the preference of cancer cells to use the glycolytic pathway still remain to be investigated, this altered energy metabolism may represent a vulnerability of cancer to therapeutic intervention (7). Similar to many other cancer types with high FDG-PET signal $(6,8)$, NPC cells seem to be highly active in glycolysis and can be readily detected using FDG-PET scan, which in fact has been used for diagnosis and prognosis evaluation of NPC with high standard uptake values (SUV) being associated with poor prognosis (9). This is consistent with the recent study suggesting that active aerobic glycolysis occur in the head and neck squamous carcinoma (10). However, it remains unclear if the active glycolytic activity in NPC cells could be exploited for therapeutic purpose and the key enzymes in the glycolytic pathway that may be targeted to kill NPC cells remain to be investigated.

Lactate dehydrogenase (LDH) is an enzyme that catalyzes the conversion of pyruvate to lactate and thus significantly affect the glycolytic metabolism. Interestingly, the expression of LDH has been found to be an independent prognosis biomarker in different cancers including NPC $(11,12)$. Furthermore, the level of LDH seemed to be associated with NPC bone metastasis (13). Knockdown of LDH has 
been shown to cause inhibition of tumor growth in vitro and in vivo in several types of human cancers (14-18), indicating an association between elevated glycolytic activity and the malignant behavior of NPC cells. These observations suggest that LDH may be a key enzyme that plays an essential role in energy metabolism in NPC cells and that it may be possible to target this enzyme as a therapeutic strategy for treatment of this malignant disease. In the present study, we showed that inhibition of LDH by oxamate led to significant suppression of glucose uptake and lactate generation and inhibited NPC growth in vitro and in vivo. We also found a drug combination strategy that is highly effective in abrogating energy metabolism in NPC cells and significant therapeutic implications.

\section{Materials and methods}

Reagents. Oxamate was purchased from Sigma-Aldrich. LDH activity detection kit was purchased from Biovision (San Francisco, CA, USA). Strips for glucose and lactate detection were the product of Roche (Mannheim, Germany). Annexin V/ PI staining reagents were purchased from Keygene (Nanjing, China). MitoSOX and Rhodamine-123 were from Molecular Probes (OR, USA). ATP detection kit was purchased from Promega (Madison, WI, USA).

Cell culture. Human nasopharyngeal carcinoma cell lines CNE1 and CNE2 were cultured in DMEM medium supplemented with $10 \%$ FBS. The immortalized nasopharyngeal epithelial cells (NP69) were cultured in keratinocyte-SFM medium (Invitrogen) supplemented with bovine pituitary extract (BD Biosciences). A hypoxic culture condition was created by incubating cells in a sealed modular incubator chamber (Billups-Rothenberg, Del Mar, CA, USA) flushed with a gas mixture containing $5 \% \mathrm{CO}_{2}$ and $95 \% \mathrm{~N}_{2}$. Because the culture flasks contained ambient oxygen at the beginning of the experiments, the final oxygen content in the hypoxia chamber was $\sim 0.5-1 \%$ after achieving air equilibrium as described previously (19).

MTT assay. Cells in logarithmic growing phase were seeded in 96-well plates with 3,000 cells in each well. Various concentrations of oxamate were added to the wells and the plates were placed in a cell culture incubator for $72 \mathrm{~h}$. The samples were then incubated with MTT reagent (20 $\mu \mathrm{l} /$ well) and incubated for another $4 \mathrm{~h}$. After the culture medium was removed, the cells were lysed with $200 \mu$ DMSO to dissolve the purple formazan and the OD values were detected at the wavelength of $570 \mathrm{~nm}$ as described previously (20).

Colony formation assay. Cells in logarithmic phase were seeded in 6-well plates with 500 cells in each well. Various concentrations of oxamate were added to the wells. The cells were cultured for two weeks before fixation and staining with crystal violet. The samples were photographed and the colonies were counted as described previously (21).

Annexin V/PI assay. Apoptosis was detected using Annexin V/ PI double staining as described previously (20). After the drug treatment, cells were harvested, washed and counted. The cells were then stained with Annexin V and PI in binding buffer for
15 min and cell viability was analyzed using the FACSCalibur flow cytometer (BD Co., USA).

Cell cycle analysis. Cells in logarithmic phase were seeded in 6 -well plates with a density of $2 \times 10^{5}$ cells per well. Different concentrations of oxamate were added and the plates were incubated for $24 \mathrm{~h}$. The cells were harvested and fixed with $75 \%$ alcohol at $4^{\circ} \mathrm{C}$ for $4 \mathrm{~h}$ and then incubated with RNase at $37^{\circ} \mathrm{C}$ for $30 \mathrm{~min}$ followed by addition of PI. Finally, cell cycle distribution were detected by using the BD FACSCalibur flow cytometer (22).

Glucose uptake and lactate production assays. Cells were seeded in 6-well plate with the density of $2 \times 10^{5}$ cells/well. After the indicated incubation periods, medium was removed for measurement of glucose and lactate concentrations using the Roche Accurend Analyzer (Roche) as previously described (23). Experiments were repeated for at least three times.

Assay of cellular ATP. Cells in logarithmic growth were seeded in 96-well opaque plates with the density of 50,000 cells per well. Oxamate $(50 \mathrm{mM})$ was added and the plates were incubated for $6,12,24$ or $48 \mathrm{~h}$. Cellular ATP contents were measured using the Promega ATP detection kits according to the procedures recommended by the manufacturer. Luminescence (relative light unit) was detected by a luminescent plate reader as previously described (23). ATP contents in the samples were calculated according to the standard curve.

Reactive oxygen species (ROS) and mitochondrial transition potential (MTP) detection. Cells were stained for $30 \mathrm{~min}$ with DCF-DA for ROS detection or Rhodamine-123 for MTP detection. The samples were harvested and analyzed using a BD Calibur flow cytometry as described previously (24).

LDH activity assay. LDH activity was analyzed as described previously (25). Briefly, cells were washed, homogenized and centrifuged. The supernatant was added into a 96-well plate. After addition of the reaction mix, enzyme reaction was initiated and OD value was detected at the wavelength of $450 \mathrm{~nm}$. LDH activity was calculated as described previously (26).

Animal study. Balb/c nude mice (age 4-6 weeks) were purchased from the Laboratory Animal Center of Guangdong Province. Animal study was performed according to a research protocol approved by the ethics committee of the laboratory animal research of Sun Yat-Sen University Cancer Center. Two million CNE-2 cells were inoculated into the right flank of the mice. When the average tumor volume reached $100 \mathrm{~mm}^{3}$, the mice were divided into two groups, a control group and the oxamate-treatment group (with 5 mice in each group). Oxamate was given by i.p. injection $(750 \mathrm{mg} / \mathrm{kg}$, daily) as described previously (25). The control group was administered with PBS. The mouse body weights and tumor volumes were measured twice weekly. At the end of the experiment, mice were sacrificed and the tumors were excised for pathological examination. The tumor volume was calculated according to the following formula: Tumor volume $\left(\mathrm{mm}^{3}\right)=a b^{2} / 2$, where $a$ and $b$ represent the long and short diameter of the tumor, respectively. 


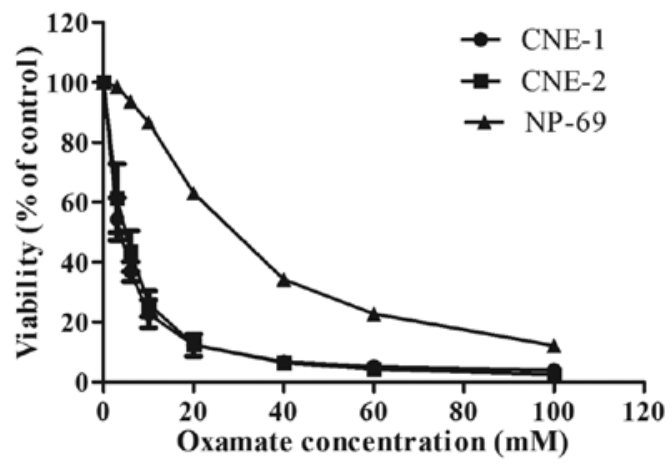

Figure 1. Effect of oxamate on cell proliferation in human nasopharyngeal carcinoma cell lines (CNE-1 and CNE-2) in comparison with immortalized human normal nasopharyngeal epithelial cells (NP-69). Cells were incubated with the indicated concentrations of oxamate for $72 \mathrm{~h}$ and cell viability was measured by MTT assay. Data are expressed as the mean \pm SD from $\geq 3$ separate experiments. A $\mathrm{P}<0.05$ is regarded as statistically significant.

Statistical analysis. Data are presented as the means and SD of at least three independent measurements. Statistical analysis (one way ANOVA or unpaired t-test) was used to determine differences between means of different experimental groups. Significance level for all statistical comparisons was set at $\mathrm{P}<0.05$. Graph pad Prism 5.0 software (US) was used to draw pictures. Canvas X (US) was used to align and process pictures.

\section{Results}

Selective anti-proliferative effect of oxamate against NPC cells. We first used MTT assay to evaluate the effect of oxamate treatment on the proliferation of two human NPC cell lines (CNE-1 and CNE-2). The results showed that oxamate caused a strong inhibition of proliferation in nasopharyngeal carcinoma cell lines in a dose-dependent manner (Fig. 1). CNE-1 and CNE-2 exhibited similar sensitivity to oxamate with the $\mathrm{IC}_{50}$ values of $3.6 \pm 0.75$ and $4.80 \pm 0.95 \mathrm{mM}$, respectively. Intriguingly, the cytotoxicity of oxamate against immortalized human normal nasopharyngeal epithelium NP-69 cells was markedly less (higher $\mathrm{IC}_{50}$ value, $30 \mathrm{mM}$ ) compared to malignant NPC cell lines $(\mathrm{P}<0.05)$, indicating a preferential inhibitory effect of oxamate against NPC cells (Fig. 1). We also used colony formation assay to further evaluate the effect of oxamate on the colony forming ability of NPC cells. The results showed that oxamate was able to effectively suppress colony formation of NPC cells in a concentration-dependent manner, with $>80 \%$ of colonies lost when treated with $18 \mathrm{mM}$ oxamate in both NPC cell lines (Fig. 2, P<0.01).
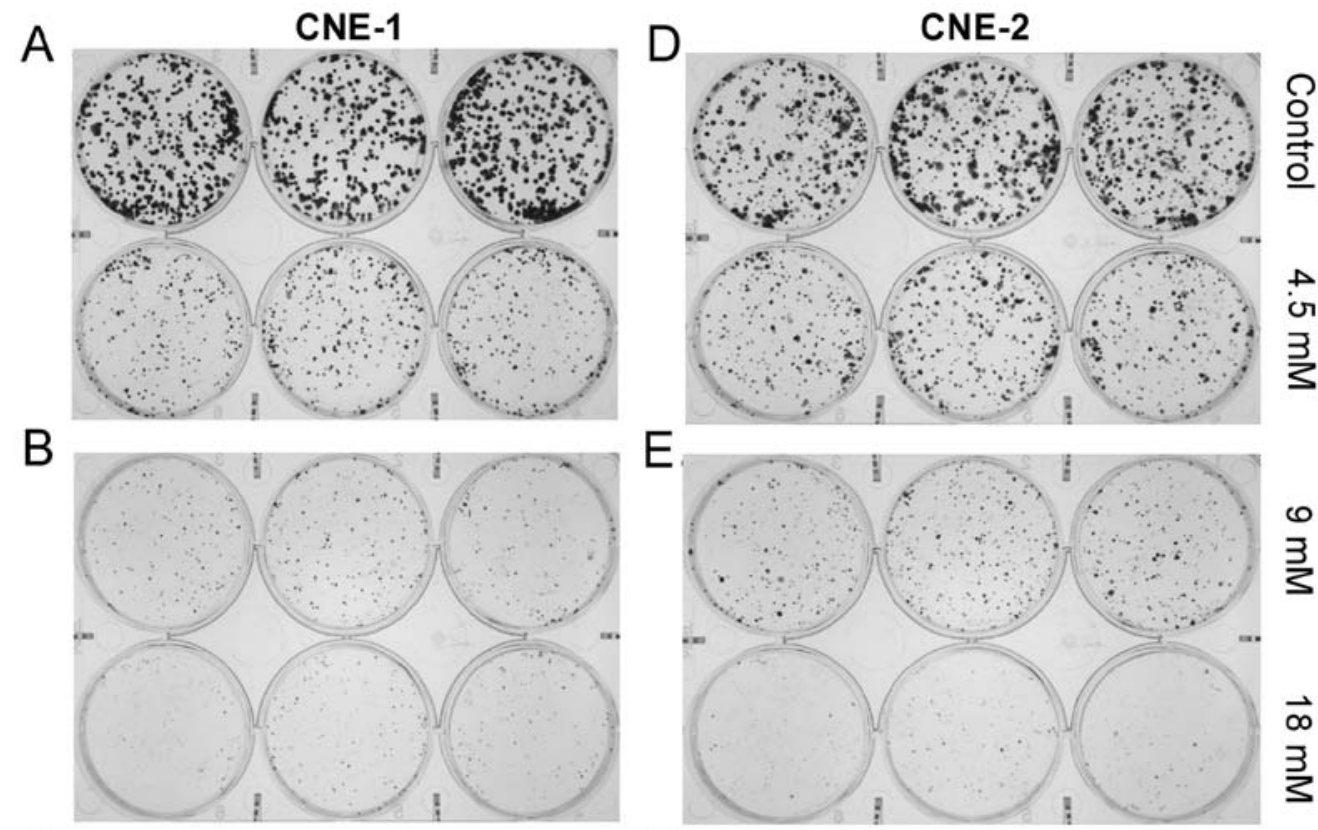

E
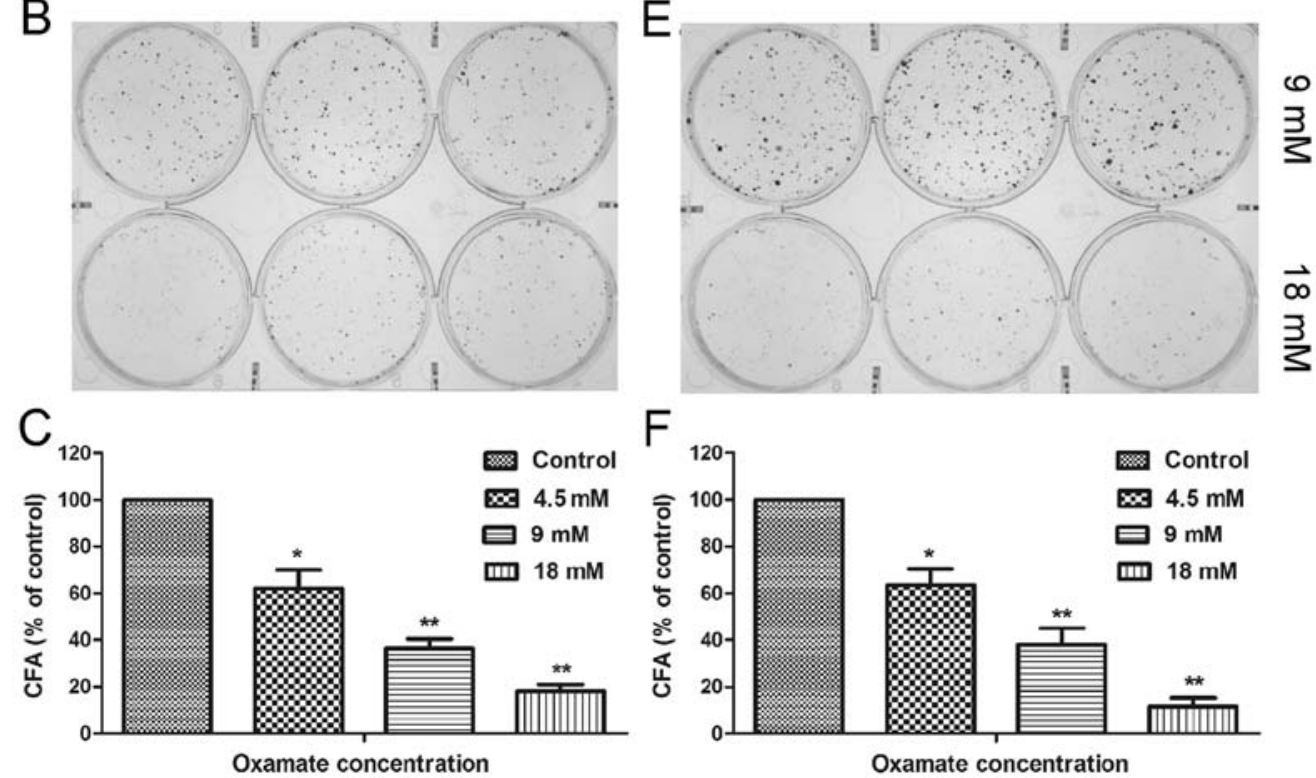

Figure 2. Inhibition of colony formation in NPC cells by oxamate. CNE-1 and CNE-2 cells were incubated with the indicated concentrations of oxamate ( 0 , 4.5, 9 and $18 \mathrm{mM}$ ) for two weeks and cell colonies with fixed, stained and counted. (A-C) Effect of oxamate on colony formation in CNE-1 cells. (D-F) Effect of oxamate on colony formation in CNE- 2 cells. Data are expressed as the mean \pm SD of $\geq 3$ experiments; a P $<0.05$ is considered statistically significant. ${ }^{*} \mathrm{P}<0.05 ;{ }^{* *} \mathrm{P}<0.01$. 
CNE-1

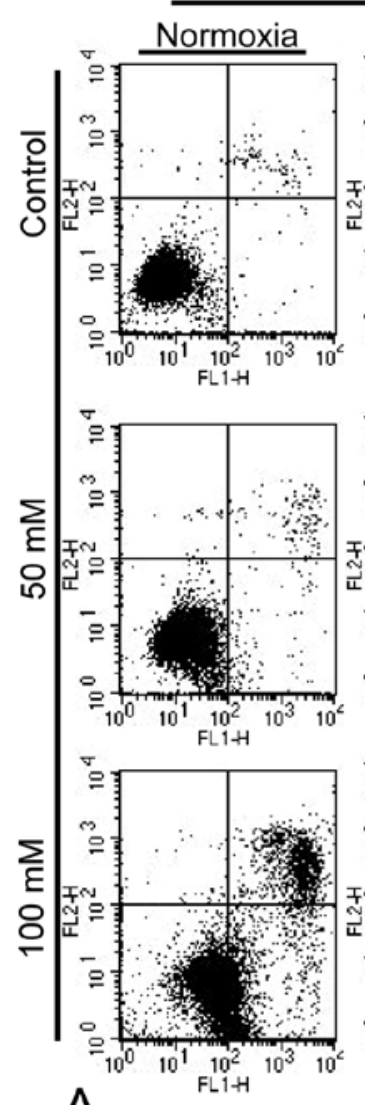

A

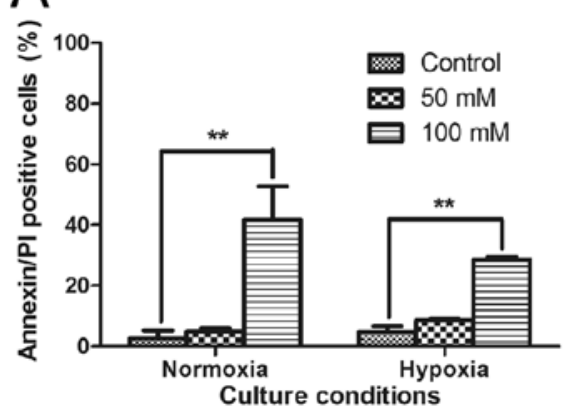

CNE-2
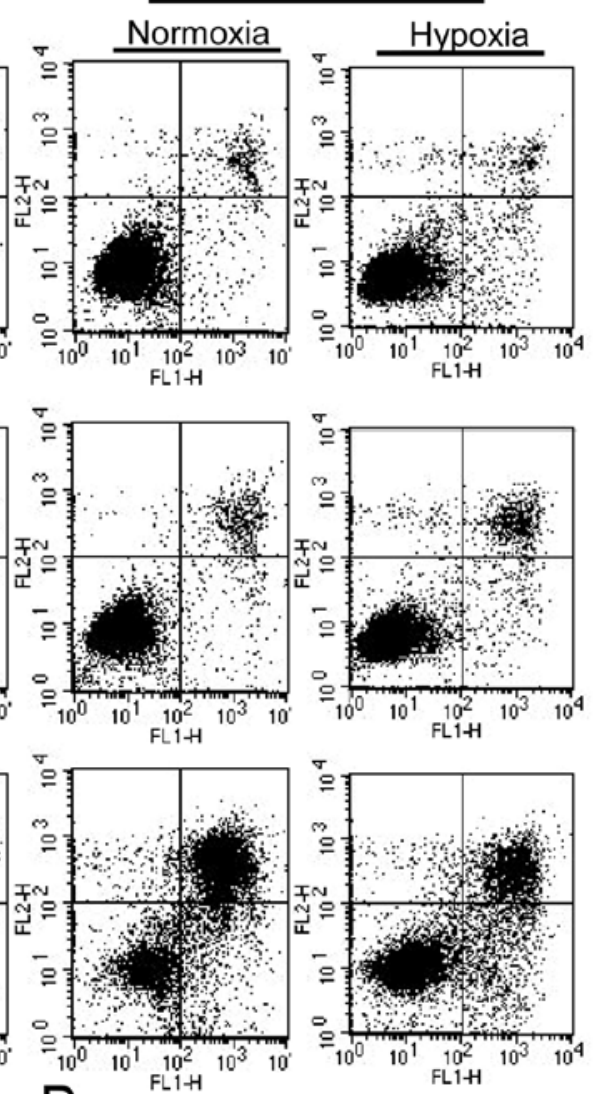

B

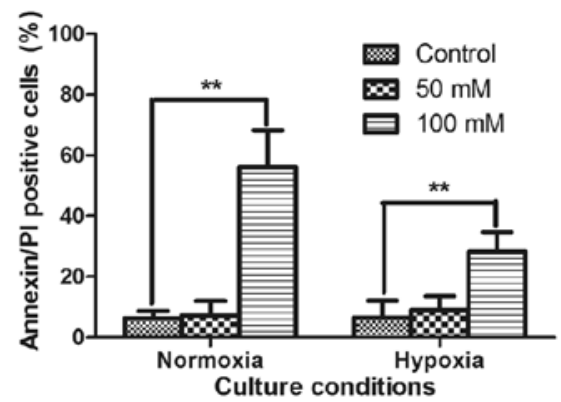

Figure 3. Cytotoxic effect of oxamate in NPC cells under normoxia and hypoxia. CNE-1 and CNE-2 cells were incubated with the indicated concentrations of oxamate $(0,50$ and $100 \mathrm{mM}$ as indicated) for $48 \mathrm{~h}$ and cell death was measured using Annexin V/PI double-staining. Data are expressed as the mean \pm SD of $\geq 3$ experiments; a $\mathrm{P}<0.05$ is considered statistically significant. ${ }^{*} \mathrm{P}<0.05 ;{ }^{* *} \mathrm{P}<0.01$.

Induction of apoptosis by oxamate in NPC cells. We then further tested if the strong anti-proliferative activity of oxamate was due to cytotoxic effect or cytostatic effect. Annexin V/PI double-staining assay was first used to test if oxamate can directly induce apoptotic cell death in NPC cells under normaxic and hypoxic conditions. The results indicated that oxamate at high concentrations (50-100 mM) was able to induce apoptosis in both CNE-1 and CNE-2 under normoxia (Fig. 3, $\mathrm{P}<0.01$ ). Interestingly, this compound seemed also effective in causing apoptosis under hypoxia (1\% oxygen), although at a slightly lower rate (Fig. 3, P<0.01).

Cell cycle analysis to test the potential effect of oxamate on cell cycle progression. As shown in Fig. 4, oxamate at the concentrations of $25-50 \mathrm{mM}$ did not significantly affect cell cycle distributions (Fig. 4C-G), while a higher concentration $(100 \mathrm{mM})$ induced cell cycle arrest at G2 phase in both CNE-1 cells (Fig. 4D) and CNE-2 cell (Fig. 4H).

Oxamate inhibits glycolytic activity in NPC. Since oxamate is an inhibitor of lactate dehydrogenase (LDH), we tested if this compound could affect glucose uptake and lactate generation, two key indicators of glycolytic activity, in NPC cells. The ability of oxamate to inhibit LDH activity was first confirmed in two NPC cell lines. As shown in Fig. 5A and B, incubation of CNE-1 and CNE-2 cells with $50 \mathrm{mM}$ oxamate resulted in a time-dependent inhibition of LDH in both cell lines $(\mathrm{P}<0.01)$. Interestingly, the basal LDH activity in CNE-2 cells (150 U) was significantly higher than that of CNE-1 (20 U), which could be a reason for the higher sensitivity of CNE-2 cells to 

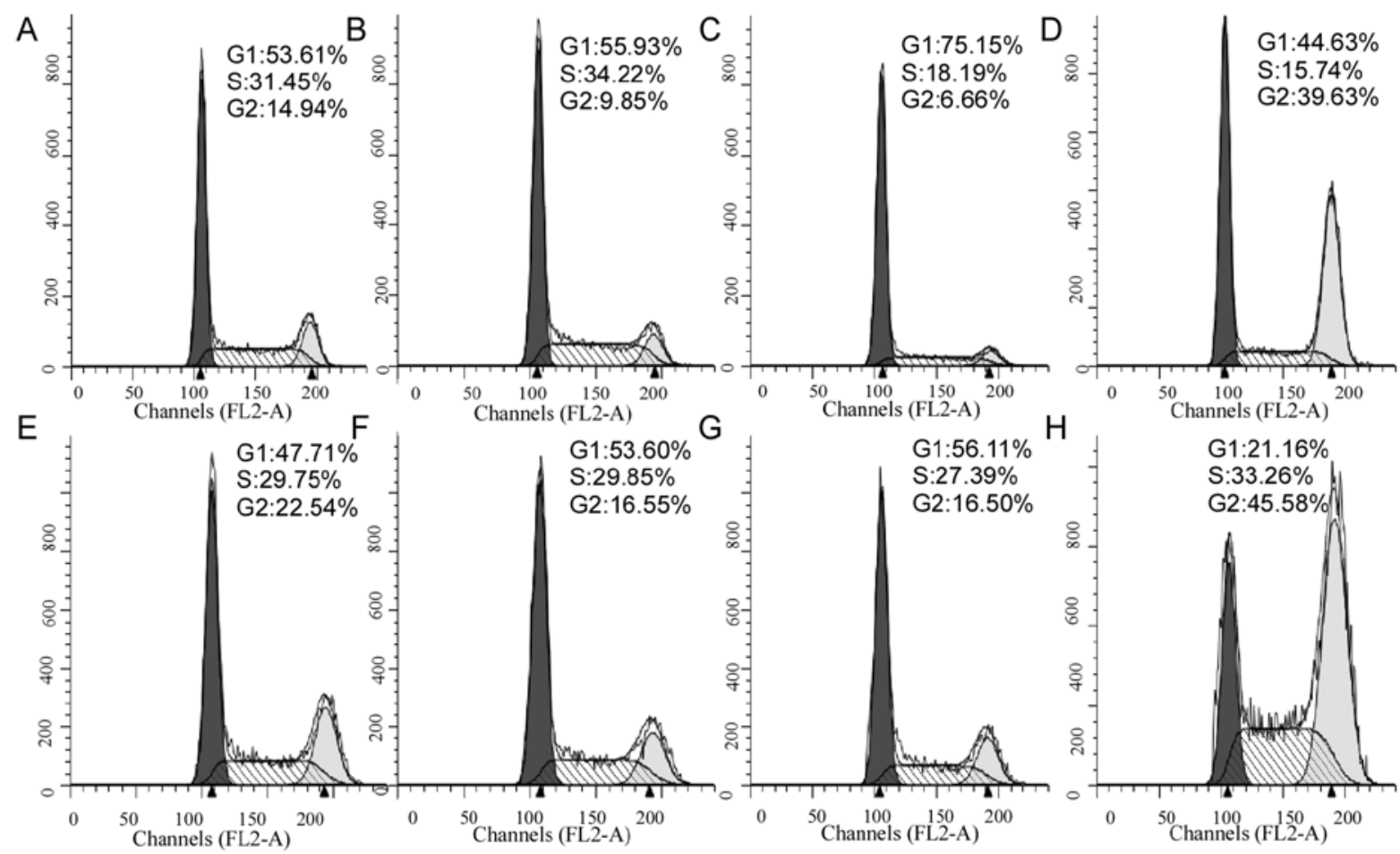

Figure 4. Effect of oxamate on cell cycle progression in nasopharyngeal carcinoma cells. CNE-1 and CNE-2 cells were incubated with different concentrations of oxamate $(0,25,50$ and $100 \mathrm{mM})$ for $24 \mathrm{~h}$ and cell cycle distributions were measured by flow cytometry. (A-D) Representative cell cycle distribution in CNE-1 cells treated with $0,25,50$ and $100 \mathrm{mM}$ oxamate. (E-H) Representative cell cycle distribution in CNE-2 cells treated with $0,25,50$ and and $100 \mathrm{mM}$ oxamate.

oxamate in apoptotic response (Fig. 3) and cell cycle arrest (Fig. 4), since this cell line might be more dependent on LDH.

To further evaluate the impact of oxamate on glucose energy metabolism in NPC cells, we analyzed the effect of this compound on glucose uptake, lactate production and cellular ATP contents. Incubation of NPC cells with oxamate caused a time-dependent decrease in glucose uptake (Fig. 5C, $\mathrm{P}<0.05$ ) and lactate production (Fig. 5D, P<0.05) in both CNE-1 and CNE-2 cell lines. Such decrease was consistent with the timedependent inhibition of LDH enzyme activity (Fig. 5A and B). Interestingly, inhibition of glycolysis by oxamate only caused a transient decrease in cellular ATP by $20-40 \%$ during the first 6-12 $\mathrm{h}$ and cellular ATP levels restore to the control levels by $24-48 \mathrm{~h}$ (Fig. $5 \mathrm{E}, \mathrm{P}>0.05$ ). These data together suggest that NCP cells were able to compensate the loss of ATP due to glycolytic inhibition by upregulation of another energy production pathway such as oxidation phosphorylation in the mitochondria.

Oxamate induces an increase in mitochondrial transmembrane potential and ROS. The potential effect of oxamate on mitochondria functional status was tested in two NPC cell lines. As shown in Fig. 6, we used two fluorescent probes the Rhodamine-123 and Mito-SOX to evaluate the effect of oxamate on mitochondrial transmembrane potential (MTP) and reactive oxygen species (ROS), respectively, and showed that treatment of NPC cells with $50 \mathrm{mM}$ oxamate induces a significant increase in MTP and ROS levels both in CNE-1 and CNE-1 cells (Fig. 6, P<0.05). These changes seem to suggest that the mitochondrial metabolic activity might be increased in response to inhibition of glycolysis in the cytosol to compensate ATP generation. This possibility was further confirmed by drug combination study in which inhibition of mitochondrial respiration by rotenone significantly enhances the effect of oxamate in terms of ATP depletion and cytotoxicity.

Inhibition of mitochondrial respiration in combination with oxamate leads to synergistic ATP depletion and cytotoxicity in NPC cells. Mitochondrial oxidative phosphorylation and glycolysis in cytosol are two major pathways that produce cellular ATP. Based on the observations that oxamate inhibited glycolysis and caused an increase in mitochondrial ROS and MTP, we postulated that glycolytic inhibition by oxamate might cause a compensatory increase in mitochondrial ATP generation, which prevented ATP depletion and massive cell death. To investigate this possibility, we used rotenone, a specific inhibitor of mitochondrial respiratory chain complex I, in combination with oxamate and tested their combined effect on cellular ATP and cell viability. As shown in Fig. 7, a sub-toxic concentration of oxamate $(3.15 \mathrm{mM})$ or a sub-toxic concentration of rotenone $(10 \mathrm{nM})$ alone did not cause any significant depletion of ATP in CNE-1 cells (Fig. 7A) or CNE-2 cells (Fig. 7B, P<0.01). Combination of both compounds at the same concentrations led to a synergistic depletion of cellular ATP and massive loss of cell viability by $>80 \%$ in both cell lines (Fig. $7 \mathrm{C}$ and $\mathrm{D}, \mathrm{P}<0.01$ ). These results together suggest that NCP cells might be able to utilize glycolysis and oxidative phosphorylation to compensate for the loss of ATP if one of these metabolic pathways is inhibited and that a simultaneous 
A

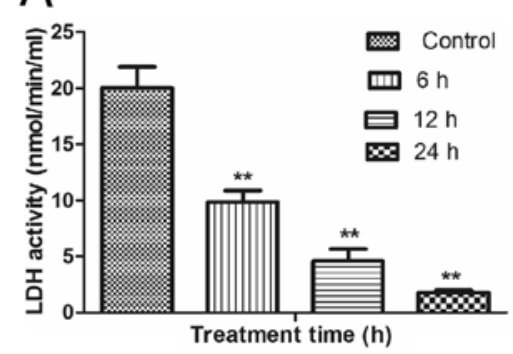

C

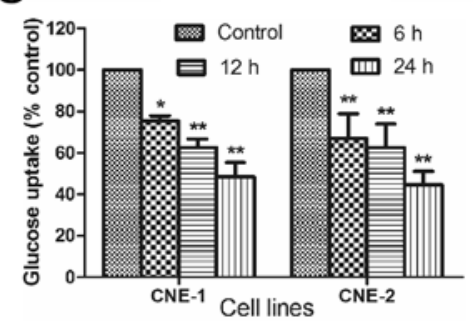

B

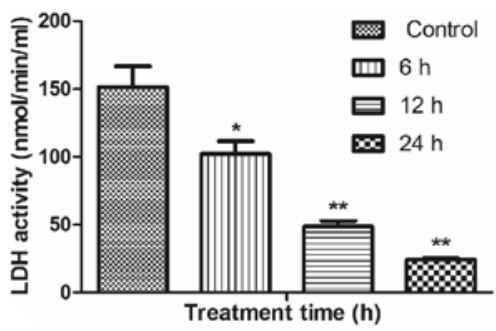

D

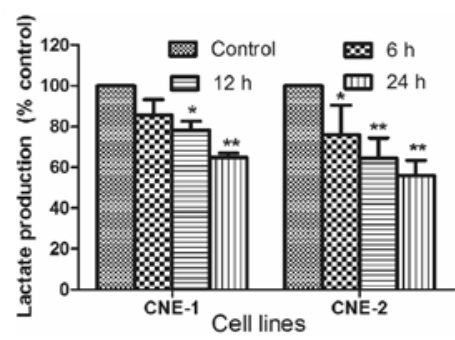

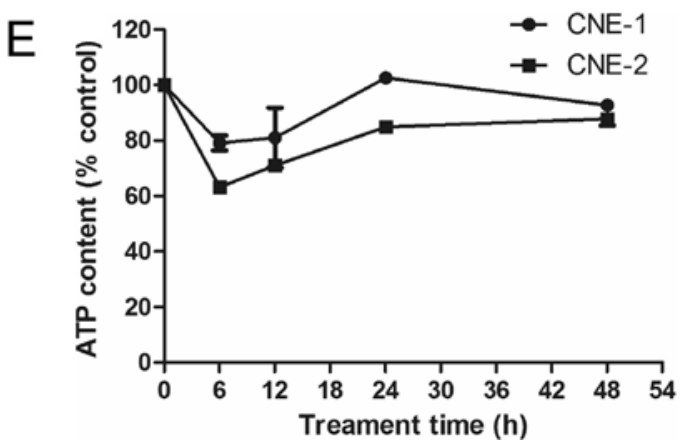

Figure 5. Effect of oxamate on the glycolytic activities in NPC cells. (A) Inhibition of LDH by oxamate in CNE-1 cells. Cells incubated with 50 mM oxamate for 6-24 h as indicated and LDH activity in the cell extracts was measured as described in Materials and methods. (B) Inhibition of LDH by oxamate in CNE-2 cells. Cells incubated with $50 \mathrm{mM}$ oxamate for 6-24 h as indicated and LDH activity in the cell extracts was measured as described in Materials and methods. (C) Effect of oxamate $(50 \mathrm{mM})$ on glucose uptake in NPC cells. (D) Effect of oxamate (50 mM) on lactate generation in NPC cells. (E) Effect of oxamate $(50 \mathrm{mM})$ on cellular ATP contents. Data are expressed as the mean \pm SD of three repeated experiments.
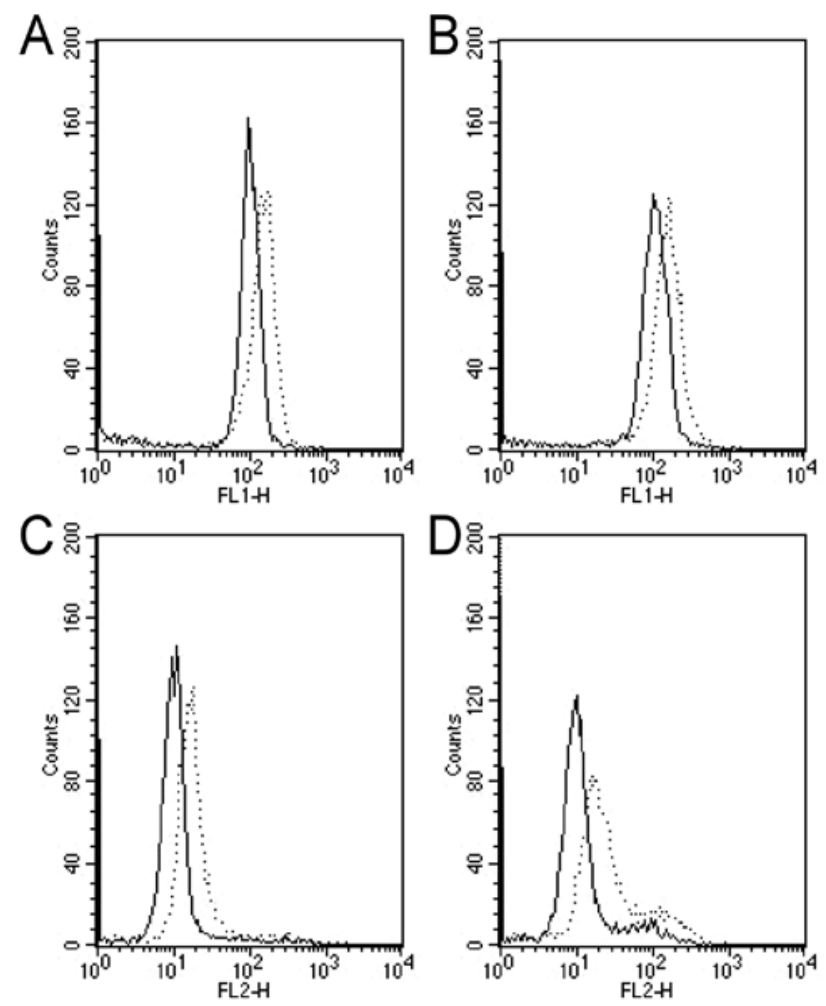

inhibition of both pathways would be highly effective in killing NPC cells.

Antitumor activity of oxamate in vivo. Animal experiments were performed to test the potential therapeutic activity of oxamate against NPC. CNE-2 cells were inoculated subcutaneously ( 2 million cells/injection) into the right flanks of nude mice. When the tumor volume reached approximately $100 \mathrm{~mm}^{3}$, the mice were divided into two groups (5 mice/each). The treatment group received oxamate injection $(750 \mathrm{mg} / \mathrm{kg}$, i.p.) daily, while the control group received PBS treatment. As shown in Fig. 8A, oxamate treatment substantially inhibited

Figure 6. Effect of oxamate on mitochondrial transmembrane potential (MTP) and reactive oxygen species (ROS) in NPC cells. CNE-1 and CNE-2 cells were incubated with $50 \mathrm{mM}$ of oxamate for $24 \mathrm{~h}$. Mitochondrial ROS was measure by MitoSOX; Mtochondrial transmembrane potential was quantified by staining with Rhodamine-123. The solid line and the dotted line in the graph represent the control group and oxamate-treated group, respectively. (A) ROS changes in CNE-1 cells treated with $50 \mathrm{mM}$ oxamate for $24 \mathrm{~h}$. (B) ROS changes in CNE-2 cells treated with $50 \mathrm{mM}$ oxamate for $24 \mathrm{~h}$. (C) MTP change in CNE-1 cells treated with $50 \mathrm{mM}$ oxamate for $24 \mathrm{~h}$. (D) MTP change in CNE-2 cells treated with $50 \mathrm{mM}$ oxamate for $24 \mathrm{~h}$. Representative data of three repeated experiments are shown. 

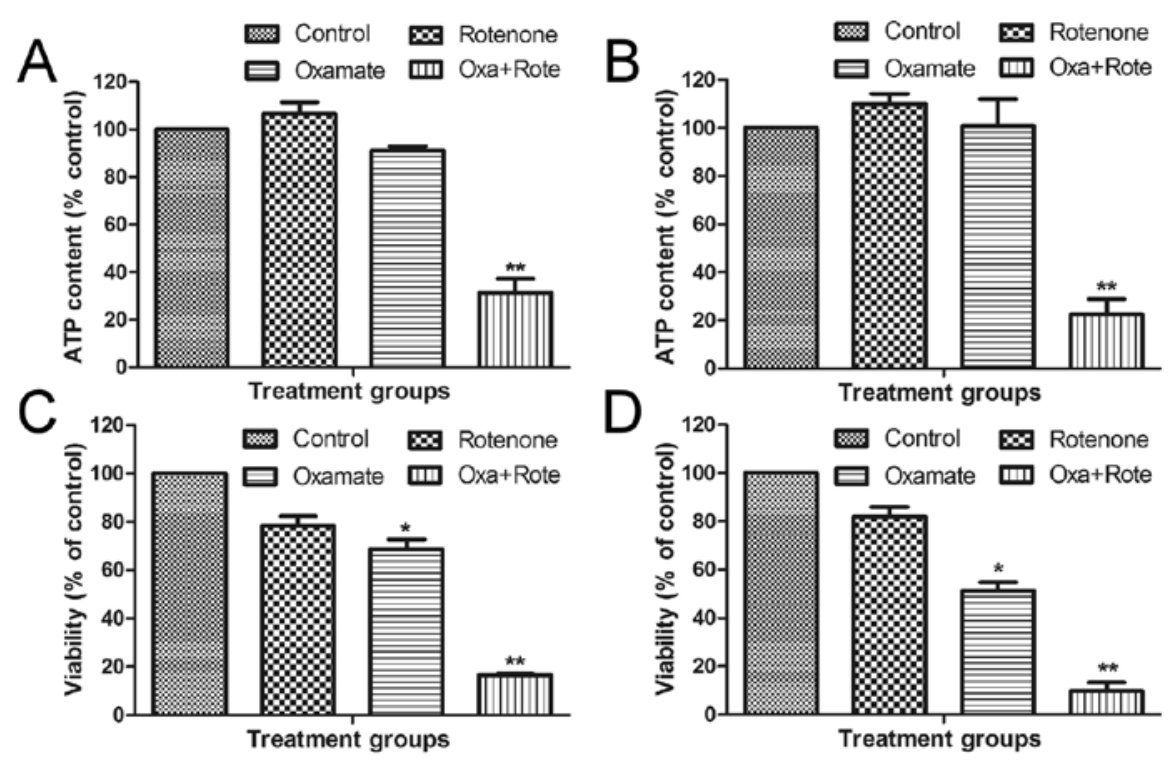

Figure 7. Effect of oxamate and rotenone on cellular ATP and viability in NPC cells. (A) CNE-1 cells were incubated with oxamate (3.15 mM), rotenone $(0.01 \mu \mathrm{M})$, or their combination for $24 \mathrm{~h}$ as indicated, ATP content was measured. (B) CNE-2 cells were incubated with oxamate (3.15 mM), rotenone $(0.01 \mu \mathrm{M})$, or their combination for $24 \mathrm{~h}$ as indicated, ATP content was measured. (C) CNE-1 cells were incubated with oxamate (3.15 mM), rotenone ( $0.01 \mu \mathrm{M})$, or their combination for $72 \mathrm{~h}$ as indicated, cell viability was then measured. (D) CNE-2 cells were incubated with oxamate $(3.15 \mathrm{mM})$, rotenone $(0.01 \mu \mathrm{M})$, or their combination for $72 \mathrm{~h}$ as indicated, cell viability was then measured; ${ }^{*} \mathrm{P}<0.05 ;{ }^{* *} \mathrm{P}<0.01$.
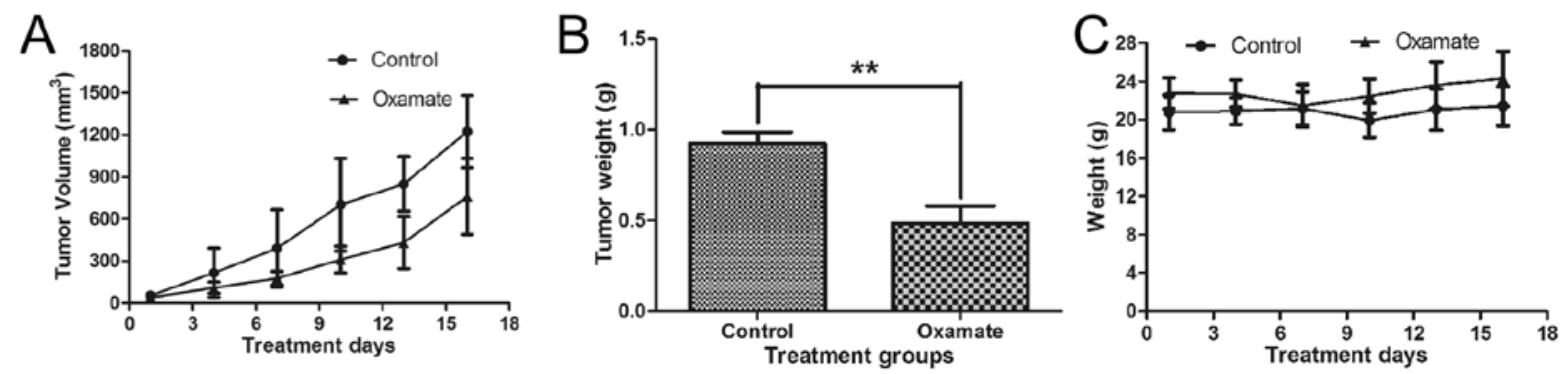

Figure 8. In vivo therapeutic activity of oxamate in NPC tumor-bearing mice. (A) Balb/c nude mice were inoculated with CNE-2 cells and divided into two groups (5 mice/group). Mice in the control group were treated with PBS, mice in the treatment group were injected with oxamate ( $750 \mathrm{mg} / \mathrm{kg}$, i.p., daily). Tumor growth was monitored by measuring the tumor size. (B) At the end of the experiment, tumors were removed and weights were compared. (C) Mouse body weights. ${ }^{* *} \mathrm{P}<0.01(\mathrm{n}=5)$.

tumor growth. At the end of the experiment, tumors were removed for weighing. The average tumor weights in the oxamate-treated group were significantly less than that of the control group $(\mathrm{P}<0.01$, Fig. $8 \mathrm{~B}, \mathrm{P}<0.01)$. The daily oxamate treatment seemed well-tolerated by the animals and there was no significant loss of body weights (Fig. $8 \mathrm{C}, \mathrm{P}>0.05$ ). These data together suggest that oxamate may have potential for treatment of NPC.

\section{Discussion}

Accumulating evidence suggests that metabolic alterations are one of the important hallmarks of cancer and that such biochemical changes may be exploited for therapeutic purpose (26). Increase in aerobic glycolysis is perhaps the most prominent metabolic alteration observed in cancer cells with potentially significant therapeutic implications in clinical cancer treatment (27). Several small molecular weight compounds such as 2-deoxyglucose, 3-bromopyruvate, loni- damine and oxamate have been shown to inhibit glycolysis with significant anticancer activity in vitro and in vivo (28). Owing to the promising anticancer effects of glycolytic inhibitors observed in cell culture and in animal tumor models, it would be important to evaluate glycolytic inhibitors in clinical setting and to further develop new generation of compounds with better therapeutic potential. In this research area, several metabolic targets and inhibitors are emerging $(29,30)$.

Among the small compounds that inhibit cancer energy metabolism, oxamate was initially identified over 40 years ago as an inhibitor of LDH (31). Inhibition of LDH by oxamate causes a decrease in lactate production and suppression of cancer cell proliferation. In our study, we showed that oxamate was able to kill NPC cells both in normoxic and hypoxic conditions (Fig. 3). This is important because many conventional anticancer drugs become less effective in hypoxia and thus compounds capable of killing cancer cells in hypoxia would potentially be useful to overcome hypoxiainduced drug resistance. Mechanistically, hypoxic cells are 
more dependent on glycolysis to generate ATP due to limited availability of oxygen for oxidative phosphorylation. As such, glycolytic inhibition is expected to be effective in killing hypoxic cells. Previous study indeed suggested that hypoxia rendered cancer cells sensitive to glycolytic inhibitors (32). It is possible, however, that induction of HIF-1 $\alpha$ by hypoxia and activation of Akt and other survival molecules may protect tumor cells from the cytotoxic effect of oxamate to some degree.

Previous study showed that high expression of LDH in breast cancer cells was associated with active glycolysis and resistance to taxol and that inhibition of $\mathrm{LDH}$ by oxamate sensitized the tumor cells to taxol (33). Interestingly, it was shown that the high expression of ErbB2 in breast cancer cells promoted LDH expression and that oxamate was able to effectively kill these breast cancer cells with Erb2 overexpression (34). Furthermore, breast cancer cells resistant to trastuzumad (a drug that suppresses Erb2 signaling) was found to overexpress LDH and inhibition of LDH by oxamate or by RNAi sensitized these cancer cells to trastuzumad (25). The important role of glycolytic inhibition in sensitization of cancer cells to chemotherapy and radiotherapy has also been demonstrated in other experimental systems $(35,36)$. These data, together with our observations that oximate was effective in killing NPC cells in vitro and suppressing NPC tumor growth in vivo, show the promising therapeutic potential of using oxamate in cancer treatment. However, it should be noted that the effective concentrations of oxamate required to kill cancer cells are very high (in $\mathrm{mM}$ range), which might limit its clinical use. The development of more effective LDH inhibitors such as Galloflavin with potent anticancer activity (37) may provide a solution.

In this study, oxamate exhibited selective cytotoxic effect against NPC cells with less cytotoxicity toward immortalized human nasopharyngeal epithelial cells (NP-69) in vitro and was well-tolerated in animals, suggesting that normal cells are less sensitive to glycolytic inhibition, likely due to their normal mitochondrial function and less dependence on glycolysis. We showed that oxamate could inhibit the glucose uptake and lactate production and that such glycolytic inhibition could be observed as early as $6 \mathrm{~h}$, which was much earlier than the appearance of apoptosis. These observations together suggest that inhibition of glycolysis was a primary event leading to cell death. In addition to glycolytic inhibition, we also observed that oxamate caused changes in mitochondria as evidenced by an increase in mitochondrial transmembrane potential and ROS generation (Fig. 6). The mechanisms responsible for oxamate-induced mitochondrial changes and their role or contributions to the cytotoxic action of oxamate remain unclear and require to further investigation. A recent study showed that oxamate was able to inhibit aspartate aminotransferase (34), suggesting that this compound may have multiple cellular targets.

Interestingly, we observed that inhibition of glycolysis by oxamate caused a short-term decrease in cellular ATP by 20-40\% during early drug exposure time period (6-12 h), but cellular ATP contents recovered to the normal level by $24-48 \mathrm{~h}$, suggesting that NPC cells (CNE-1 and CNE-2) were able to compensate the loss of ATP due to glycolytic suppression by oxamate. The mechanism responsible for this metabolic compensation was likely through activation of mitochondrial oxidative phosphorylation, since inhibition of mitochondrial respiratory chain by rotenone in combination with oxamate caused a severe depletion of cellular ATP and massive loss of cell viability (Fig. 7). These data suggest that such mechanismbased drug combination might have potential to improve therapeutic activity in NPC treatment. This is consistent with the recent findings that a combination of mitochondria-targeted drugs and the glycolytic inhibitor 2-deoxyglucose exhibit synergistic effect against breast cancer cells (38).

In conclusion, we showed that oxamate was able to inhibit glucose uptake and lactate generation in NPC cells. This compound exhibited significant anticancer activity against NPC cells in vitro under normoxia and hypoxia and suppressed tumor growth in vivo. Activation of mitochondrial oxidative phosphorylation seems to be a compensatory mechanism that may compromise the ability of oxamate to kill NPC cells. Combination of oxamate with an inhibitor of mitochondrial respiration showed strong synergistic activity. It is important to further test the promising therapeutic activity of this novel drug combination in animals. It is also critical to test the potential cytotoxic effect of such drug combination in normal cells and in animal toxicology study to evaluate the therapeutic selectivity of this novel drug combination.

\section{Acknowledgements}

The authors thank Mingli Peng of Sun Yat-Sen University Cancer Centre for assistance with mouse husbandry. This study was supported in part by a grant from the major science and technology project of the National Basic Research Program of China (973 Program grant no. 2012CB967004).

\section{References}

1. Huang TR, Zhang SW, Chen WQ, et al: Trends in nasopharyngeal carcinoma mortality in China, 1973-2005. Asian Pac J Cancer Prev 13: 2495-2502, 2012

2. Cheng SH, Jian JJ, Tsai SY, et al: Prognostic features and treatment outcome in locoregionally advanced nasopharyngeal carcinoma following concurrent chemotherapy and radiotherapy. Int J Radiat Oncol Biol Phys 41: 755-762, 1998.

3. Toh HC, Ha TC and Wee J: Personalised medicine in nasopharyngeal cancer. Lancet Oncol 13: 568-569, 2012.

4. Ayyanathan K, Kesaraju S, Dawson-Scully K and Weissbach H: Combination of sulindac and dichloroacetate kills cancer cells via oxidative damage. PLoS One 7: e39949, 2012.

5. Warburg O: On the origin of cancer cells. Science 123: 309-314, 1956.

6. Jadvar H: Imaging evaluation of prostate cancer with (18)F-fluorodeoxyglucose PET/CT: utility and limitations. Eur J Nucl Med Mol Imaging 40 (Suppl 1): 5-10, 2013.

7. Schulze A and Harris AL: How cancer metabolism is tuned for proliferation and vulnerable to disruption. Nature 491: 364-373, 2012.

8. Erdem V, Selimoglu Sen H, Komek H, et al: Prognostic factors in non-small cell lung cancer patients and prognostic importance of PET/CT SUV max value. Tuberk Toraks 60: 207-217, 2012 (In Turkish).

9. Chan WK, Kwong DL, Yeung DW, Huang B and Khong PL: Prognostic impact of standardized uptake value of F-18 FDG PET/CT in nasopharyngeal carcinoma. Clin Nucl Med 36: 1007-1011, 2011.

10. Sandulache VC, Ow TJ, Pickering CR, et al: Glucose, not glutamine, is the dominant energy source required for proliferation and survival of head and neck squamous carcinoma cells. Cancer 117: 2926-2938, 2011. 
11. Zhou GQ, Tang LL, Mao YP, et al: Baseline serum lactate dehydrogenase levels for patients treated with intensity-modulated radiotherapy for nasopharyngeal carcinoma: a predictor of poor prognosis and subsequent liver metastasis. Int $\mathbf{J}$ Radiat Oncol Biol Phys 82: e359-365, 2012.

12. Brown JE, Cook RJ, Lipton A and Coleman RE: Serum lactate dehydrogenase is prognostic for survival in patients with bone metastases from breast cancer: a retrospective analysis in bisphosphonate-treated patients. Clin Cancer Res 18: 6348-6355, 2012.

13. Zen HG, Jame JM, Chang AY, et al: Nasopharyngeal carcinoma with bone marrow metastasis. Am J Clin Oncol 14: 66-70, 1991

14. Fantin VR, St-Pierre J and Leder P: Attenuation of LDH-A expression uncovers a link between glycolysis, mitochondrial physiology, and tumor maintenance. Cancer Cell 9: 425-434, 2006.

15. Kim SH and Lee GM: Down-regulation of lactate dehydrogenase-A by siRNAs for reduced lactic acid formation of Chinese hamster ovary cells producing thrombopoietin. Appl Microbiol Biotechnol 74: 152-159, 2007.

16. Sheng SL, Liu JJ, Dai YH, Sun XG, Xiong XP and Huang G: Knockdown of lactate dehydrogenase A suppresses tumor growth and metastasis of human hepatocellular carcinoma. FEBS J 279: 3898-3910, 2012.

17. Yao F, Zhao T, Zhong C, Zhu J and Zhao H: LDHA is necessary for the tumorigenicity of esophageal squamous cell carcinoma. Tumour Biol 34: 25-31, 2013.

18. Zhang Y, Zhang X, Wang X, et al: Inhibition of LDH-A by lentivirus-mediated small interfering RNA suppresses intestinaltype gastric cancer tumorigenicity through the downregulation of Oct4. Cancer Lett 321: 45-54, 2012.

19. Xu RH, Pelicano H, Zhou Y, et al: Inhibition of glycolysis in cancer cells: a novel strategy to overcome drug resistance associated with mitochondrial respiratory defect and hypoxia. Cancer Res 65: 613-621, 2005.

20. Tang Z, Yuan S, Hu Y, et al: Over-expression of GAPDH in human colorectal carcinoma as a preferred target of 3-bromopyruvate propyl ester. J Bioenerg Biomembr 44: 117-125, 2012.

21. Lu W, Hu Y, Chen G, et al: Novel role of NOX in supporting aerobic glycolysis in cancer cells with mitochondrial dysfunction and as a potential target for cancer therapy. PLoS Biol 10: e1001326, 2012.

22. Zeng ZL, Wu MW, Sun J, et al: Effects of the biological clock gene Bmall on tumour growth and anti-cancer drug activity. J Biochem 148: 319-326, 2010.

23. Hu Y, Lu W, Chen G, et al: K-ras(G12V) transformation leads to mitochondrial dysfunction and a metabolic switch from oxidative phosphorylation to glycolysis. Cell Res 22: 399-412, 2012.

24. Chen G, Chen Z, Hu Y and Huang P: Inhibition of mitochondrial respiration and rapid depletion of mitochondrial glutathione by beta-phenethyl isothiocyanate: mechanisms for anti-leukemia activity. Antioxid Redox Signal 15: 2911-2921, 2011.
25. Zhao Y, Liu $\mathrm{H}$, Liu Z, et al: Overcoming trastuzumab resistance in breast cancer by targeting dysregulated glucose metabolism. Cancer Res 71: 4585-4597, 2011.

26. Hammoudi N, Ahmed KB, Garcia-Prieto C and Huang P: Metabolic alterations in cancer cells and therapeutic implications. Chin J Cancer 30: 508-525, 2011.

27. Chen Z, Lu W, Garcia-Prieto C and Huang P: The Warburg effect and its cancer therapeutic implications. J Bioenerg Biomembr 39: 267-274, 2007.

28. Fanciulli M, Bruno T, Giovannelli A, et al: Energy metabolism of human LoVo colon carcinoma cells: correlation to drug resistance and influence of lonidamine. Clin Cancer Res 6: 1590-1597, 2000.

29. Sun W, Liu Y, Glazer CA, et al: TKTL1 is activated by promoter hypomethylation and contributes to head and neck squamous cell carcinoma carcinogenesis through increased aerobic glycolysis and HIF1alpha stabilization. Clin Cancer Res 16: 857-866, 2010.

30. Seo M, Kim JD, Neau D, Sehgal I and Lee YH: Structure-based development of small molecule PFKFB3 inhibitors: a framework for potential cancer therapeutic agents targeting the Warburg effect. PLoS One 6: e24179, 2011.

31. Elwood JC: Effect of oxamate on glycolysis and respiration in sarcoma 37 ascites cells. Cancer Res 28: 2056-2060, 1968.

32. Liu H, Savaraj N, Priebe W and Lampidis TJ: Hypoxia increases tumor cell sensitivity to glycolytic inhibitors: a strategy for solid tumor therapy (Model C). Biochem Pharmacol 64: 1745-1751, 2002.

33. Zhou M, Zhao Y, Ding Y, et al: Warburg effect in chemosensitivity: targeting lactate dehydrogenase-A re-sensitizes taxol-resistant cancer cells to taxol. Mol Cancer 9: 33, 2010.

34. Zhao YH, Zhou M, Liu H, et al: Upregulation of lactate dehydrogenase A by ErbB2 through heat shock factor 1 promotes breast cancer cell glycolysis and growth. Oncogene 28: 3689-3701, 2009.

35. Liu H, Hu YP, Savaraj N, Priebe W and Lampidis TJ: Hypersensitization of tumor cells to glycolytic inhibitors. Biochemistry 40: 5542-5547, 2001

36. Aghaee F, Pirayesh Islamian J and Baradaran B: Enhanced radiosensitivity and chemosensitivity of breast cancer cells by 2-deoxy-d-glucose in combination therapy. J Breast Cancer 15: 141-147, 2012.

37. Manerba M, Vettraino M, Fiume L, et al: Galloflavin (CAS 568-80-9): a novel inhibitor of lactate dehydrogenase. Chem Med Chem 7: 311-317, 2012.

38. Cheng G, Zielonka J, Dranka BP, et al: Mitochondria-targeted drugs synergize with 2-deoxyglucose to trigger breast cancer cell death. Cancer Res 72: 2634-2644, 2012. 